\section{Cibler les cellules sénescentes}

\section{Une révolution dans}

le traitement des pathologies ostéo-articulaires

$>$ Avec le vieillissement des populations, nos sociétés doivent faire face à l'émergence d'un nombre exponentiel de patients atteints de maladies chroniques dégénératives liées à l'âge, telles que l'arthrose ou l'ostéoporose. Le «mieux 》vieillir sera ainsi au centre des prochains défis médicaux par un maintien de l'autonomie des sujets âgés et une réduction des coûts des services de santé. Au cours des 5 dernières années, en se fondant sur des modèles murins innovants ou des études in vitro, de nombreuses équipes ont démontré que plusieurs pathologies dégénératives liées à l'âge avaient en commun une accumulation délétère de cellules dites sénescentes. Sous le concept de sénolyse, il a ainsi été proposé d'éliminer pharmacologiquement ces cellules afin de retarder l'émergence de ces pathologies chroniques du sujet âgé. Nous nous proposons ici de faire le point sur les stratégies récemment mises en place, permettant l'identification de composés sénolytiques, ainsi que de définir leurs utilisations thérapeutiques comme traitement de l'arthrose et de l'ostéoporose. <

Le concept de sénescence cellulaire : une vieille histoire à la mode

Décrite et conceptualisée par Hayflick et Moorhead dans les années 1960, la sénescence cellulaire se résume en un syndrome cellulaire caractérisé par une sortie robuste du cycle cellulaire associé à un sécrétome particulier [1]. Elle peut être induite par des signaux intrinsèques, comme l'érosion des télomères, mais aussi en réponse à des stimulus extrinsèques inappropriés tels que les radicaux libres, l'exposition chronique à des cytokines inflammatoires ou à des facteurs trophiques [2]. La sénescence est associée

Vignette (Photo @ Inserm - Marie-Christine Hobatho).

\author{
Yassin Tachikart $^{1^{*}}$, Olivier Malaise ${ }^{1,2^{*}}$, \\ Michaël Constantinides ${ }^{1}$, Christian Jorgensen ${ }^{1,3}$, \\ Jean-Marc Brondello ${ }^{1}$
}

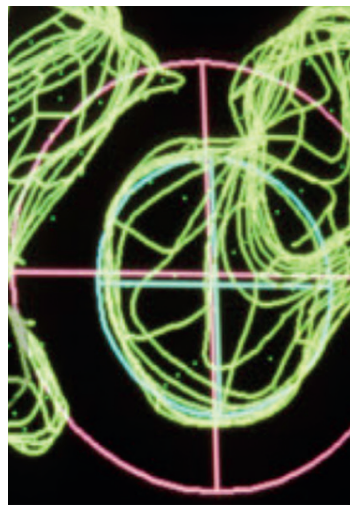

${ }^{1}$ IRMB (institut de médecine régénératrice et de biothérapie de Montpellier), Inserm U1183, Univ Montpellier, 80, avenue Augustin Fliche, 34298 Montpellier, France. ${ }^{2}$ GIGA Research (grappe interdisciplinaire de génoprotéomique appliquée), CHU de Liège et Université de Liège, Liège, Belgique.

${ }^{3}$ Service de rhumatologie, $\mathrm{CHU}$ La Peyronie, 34090 Montpellier, France.

à des changements de morphologie cellulaire, qui devient ainsi hypertrophique, à des adaptations méta-

*co-premiers auteurs

jean-marc.brondello@inserm.fr boliques, à des modifications épigénétiques, et à une signature génique particulière [3]. Plusieurs inhibiteurs du cycle cellulaire ${ }^{1}$ comme les protéines $\mathrm{p} 16^{\mathrm{INK} 4 \mathrm{a}}$ ou $\mathrm{p} 21^{\mathrm{wafl}}$, contrôlant l'activité des kinases dépendantes des cyclines, participent à l'arrêt total de la prolifération. Le programme de sénescence va ainsi induire des changements dans les fonctions de la cellule touchée tout en poussant à l'établissement d'un phénotype sécrétoire spécifique appelé SASP pour «phénotype sécrétoire associé à la sénescence », qui influence alors l'homéostasie du tissu environnant par la production de métallo-protéinases et de facteurs inflammatoires et/ou de croissance [4]. Plusieurs sous-types de sénescence cellulaire semblent exister, selon la cellule concernée ou les modes d'induction $[5,6]$. La sénescence peut ainsi être considérée comme un syndrome cellulaire plutôt qu'un phénotype unique transposable à toutes les cellules dites sénescentes. Néanmoins, des marqueurs communément retrouvés tels que l'expression de la beta-galactosidase acide ou d'inhibiteurs du cycle cellulaire, ont permis de révéler in vivo la présence de ces cellules dans de nombreux tissus et cela tout le long de l'ontogénèse [5]. Ainsi, au cours de l'établissement du placenta, par sa présence transitoire, la sénescence favorise l'implantation de l'embryon. Elle joue également un rôle dans la formation des

${ }^{1} \mathrm{pl} 6^{\text {INK4a }}$ correspond à la protéine pl6 également appelée INK4a (cyclin-dependent kinase 4 inhibitor A). De même, $\mathrm{p} 21^{\text {wafl }}$ correspond à la protéine également nommée wafl (wild-type P53-activated fragment 1 ). 
membres au cours de l'embryogenèse [5]. Par ailleurs, tout au long de la vie, la sénescence a une fonction suppressive de tumeurs essentielle en bloquant la prolifération de cellules pré-néoplasiques ayant acquis une instabilité génétique $[2,37](\rightarrow)$. $(\rightarrow)$ Voir la Synthèse de $\varepsilon$. Goy et C. Abbadie, $m / s n^{\circ} 3$, mars 2018, page 223
Elle peut également intervenir comme mécanisme

de régulation de la réponse immunitaire et participe à la différenciation terminale de certains sous-types cellulaires rares tels que les mégacaryocytes [7]. Enfin, elle favorise la réparation tissulaire comme la cicatrisation, chez les mammifères [8], et la régénération, chez la salamandre [9].

\section{Accumulation de cellules sénescentes au cours du vieillissement}

À côté de ces nombreux rôles positifs, il a été observé chez de nombreuses espèces, que les cellules sénescentes s'accumulent avec l'âge, dans des tissus mitotiques ou post-mitotiques [5]. Cette accumulation pourrait être le résultat des signaux intrinsèques ou extrinsèques itératifs qui agissent en continu sur l'homéostasie des tissus, tout au long de la vie. On retrouve ainsi une présence chronique de cellules sénescentes dans les tissus cartilagineux, osseux ou musculaire du sujet âgé, en lien avec l'émergence de pathologies dégénératives telles que, respectivement, l'arthrose, l'ostéoporose ou la sarcopénie [1013]. En dehors du système musculo-squelettique, l'accumulation de cellules sénescentes dans le tissu adipeux, l'intima artérielle, le rein vieillissant ou le poumon, a été reliée à, respectivement, la lipodystrophie du sujet âgé, l'athérosclérose, ou des insuffisances rénales et respiratoires [10-14]. En outre, cette accumulation de cellules sénescentes pourrait participer à l'émergence de ces différentes pathologies du sujet âgé en diminuant les capacités de renouvellement et de réparation des organes ciblés, tout en créant un environnement procatabolique et pro-inflammatoire par l'intermédiaire du SASP sécrété. Bien qu'attractif, il restait néanmoins nécessaire de démontrer le véritable lien causal entre l'accumulation de cellules sénescentes et une perte de fonction des tissus, puis des organes chez le sujet âgé.

Cette démonstration a été rendue possible grâce à l'utilisation d'animaux transgéniques pour des biosenseurs spécifiques de la sénescence. Un premier modèle murin appelé pl6-3MR (tri-modal reporter) consiste, sous le contrôle du promoteur du gène codant $p 16^{\text {INK4a }}$, à exprimer le gène rapporteur de la luciférase fusionné à une forme tronquée de la thymidine kinase du virus herpès simplex 1 . En présence de ganciclovir, un antiviral ciblant les herpès virus, les cellules exprimant cette thymidine kinase meurent rapidement. Le second modèle, appelé INK-ATTAC (INK-linked apoptosis through targeted activation of caspase) [38] $(\rightarrow)$, consiste en l'expression sous le contrôle du promoteur du gène codant $p 16^{\text {INK4a }}$, de la luciférase et du gène

$\rightarrow$ Voir la Nouvelle de V. Chauvet et al., $\mathrm{m} / \mathrm{s}$ $n^{\circ} 11$, novembre 2016, page 1030

« suicide » de la caspase 8 , activable après addition de la molécule AP20187 (B/B homodimerizer). Ces deux modèles permettent à la fois la détection des cellules sénescentes dans l'animal anesthésié, sous caméra CCD (charge-coupled device) en présence de luciférine, et leur élimination sélective par modulation pharmacologique. La première démonstration d'un effet bénéfique de cette élimination spécifique des cellules sénescentes, dite sénolyse, a été réalisée sur le modèle murin de vieillissement prématuré (souris $B u b R 1^{H / H}$ ) dont les souris expriment une forme hypomorphique de $B u b R l^{2}$, une kinase du contrôle de l'assemblage du fuseau mitotique. Ces souris développent, entre autres, cataracte, sarcopénie, arythmies cardiaques, et ont une espérance de vie très courte [15]. Néanmoins, un croisement de ces animaux avec les souris du modèle INK-ATTAC a permis l'induction de la sénolyse des cellules sénescentes par administration d'AP20187, entraînant un retard de l'apparition de ces pathologies et une augmentation de la longévité des souris [15].

\section{Lien causal entre sénescence cellulaire et pathologies ostéo-articulaires du sujet âgé}

L'arthrose est la plus fréquente des maladies dégénératives chroniques articulaires du sujet âgé. Plus d'un tiers de la population âgée de plus de 60 ans présente des signes radiographiques d'arthrose [16] (Figure 1). Cette pathologie conduit à des incapacités de travail prolongées, un isolement social et une dégradation de la qualité de vie du patient atteint. Plusieurs structures constituant l'articulation jouent un rôle dans l'étiologie de l'arthrose: le cartilage, mais également la membrane synoviale ${ }^{3}$. Cette membrane, qui a une fonction nourricière, va acquérir un phénotype inflammatoire et hypertrophique, contribuant alors à la sévérité des symptômes, à la formation d'ostéophytes ${ }^{4}$ et même à l'érosion articulaire [17]. De même, le remodelage de l'os sous-chondral, situé sous le cartilage articulaire, est non seulement une conséquence, mais aussi un composant actif de la maladie, générant des douleurs et une perte de la fonction articulaire [18]. Récemment, Jeon et al., en utilisant les modèles murins transgéniques pl6-3MR et INK-ATTAC, ont pu établir un lien entre la pathologie de l'arthrose et la présence de sénescence cellulaire articulaire [10]. Dans un premier temps, une arthrose expérimentale induite a été réalisée sur des souris pl6-3MR âgées de deux mois, par section du ligament croisé antérieur d'un des deux genoux. L'injection intra-articulaire de luciférine a

\footnotetext{
${ }^{2}$ Les souris BubRl ${ }^{\mathrm{H} / \mathrm{H}}$ expriment une faible quantité de la protéine BubRl (budding uninhibited by benzimidazole-related 1 ) en raison de l'introduction d'un site d'épissage alternatif au niveau de l'ARN.

${ }^{3}$ Membrane qui tapisse l'intérieur de la capsule des articulations mobiles. La synoviale est entourée par la capsule articulaire et des ligaments. Elle contient et produit la synovie, qui facilite le glissement des surfaces articulaires.

${ }^{4}$ Production osseuse anormale, formant une excroissance pouvant être localisée à proximité d'un os pathologique ou près de n'importe quelle articulation malade.
} 


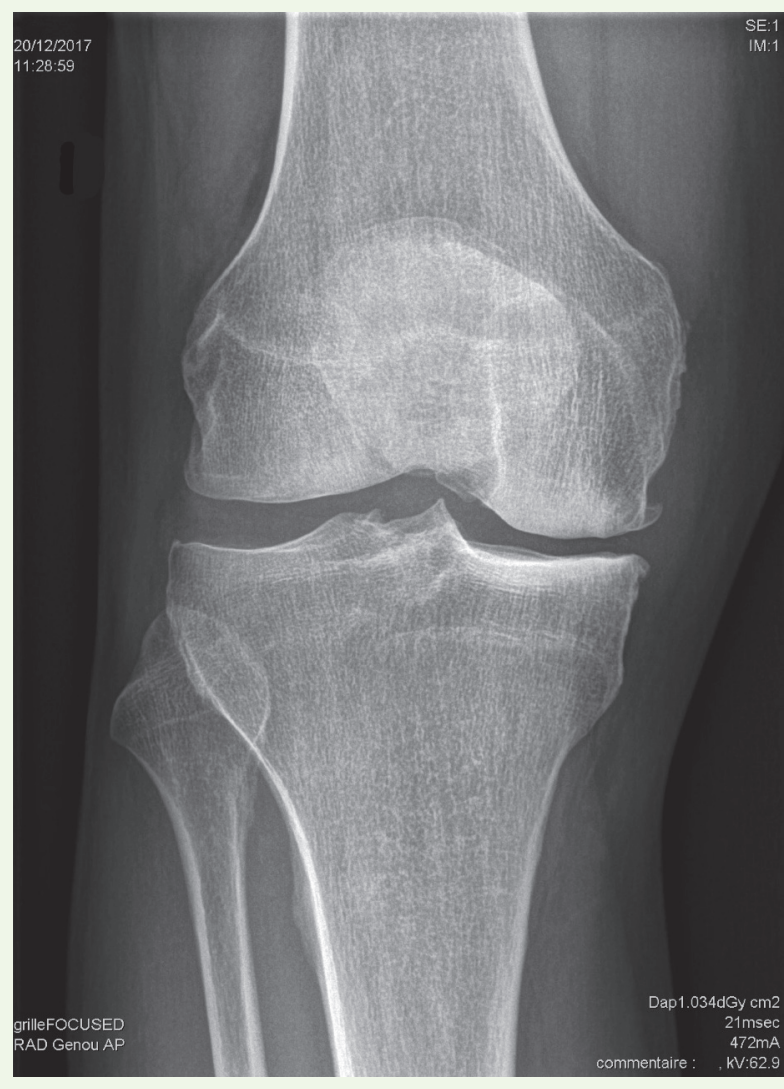

Figure 1. Radiographie de genoux montrant la perte d'épaisseur de cartilage avec début de pincement (à droite) par rapport à la zone saine (à gauche).

révélé une accumulation transitoire de cellules sénescentes à 14 jours après la chirurgie. L'analyse des genoux arthrosiques, par immunomarquage, a également permis de détecter des cellules sénescentes dans la synoviale activée et dans les zones superficielles du cartilage. Afin de déterminer si ces cellules sénescentes articulaires jouent un rôle actif dans l'apparition de l'arthrose, des injections intra-articulaires de ganciclovir ont été réalisées dans le genou des animaux ayant subi la chirurgie. De façon remarquable, l'élimination locale des cellules sénescentes s'est avérée être associée à une moindre dégradation du cartilage, une moindre expression articulaire de molécules inflammatoires telles que I'IL(interleukine)- $1 \beta$ et à une diminution de l'expression des molécules cataboliques hypertrophiques comme MMP-13 (matrix metalloproteinase-13, ou collagenase-3). L'un des principaux intérêts de cette étude était de trouver, chez les souris traitées, un niveau d'expression plus élevé de deux marqueurs prochondrogéniques, à savoir le collagène de type $l l \alpha l$ et l'aggrécane ${ }^{5}$. Pour apporter un autre argument convaincant, les auteurs ont utilisé le second modèle transgénique INK-ATTAC afin d'étudier l'apparition spontanée de l'arthrose chez des souris âgées. Fait remarquable, le traitement par AP20187, administré à ces souris INK-ATTAC dès

${ }^{5}$ Assemblage de protéoglycanes. L'aggrécane représente $10 \%$ du poids sec du cartilage animal. douze mois et jusqu'à leur mort naturelle, maintient un cartilage sain, réduisant ainsi l'apparition de signes d'arthrose spontanée liée à l'âge.

L'ostéoporose est l'autre pathologie des sujets âgés très fréquemment rencontrée dans les salles d'attente des rhumatologues. II s'agit d'une maladie du squelette caractérisée par une perte de la masse osseuse et une perturbation de la micro-architecture osseuse, augmentant le risque de fracture chez le sujet âgé. Une femme post-ménopausée sur trois en sera atteinte et, dans $40 \%$ des cas, présentera une fracture ostéoporotique [19]. L'ostéoporose est également une maladie grave: après une fracture du col du fémur chez une femme de plus de 65 ans, le risque de décès à $l$ an est en effet de $20 \%$ [20]. La prise en charge thérapeutique de l'ostéoporose pose aujourd'hui problème. Les médicaments inhibiteurs de la résorption osseuse, comme les bisphosphonates ou le denosumab ${ }^{6}$, peuvent être responsables d'ostéonécroses de la mâchoire ou de fractures fémorales atypiques [21, 22]. De plus, si ces médicaments sont assez efficaces pour prévenir une fracture de colonne, leur efficacité est plus faible au niveau de la hanche ou du poignet. Enfin, le seul traitement ostéoformateur commercialisé, le teriparatide, souffre de critères de remboursement drastiques, ce qui rend difficile sa prescription en pratique quotidienne, et le ranelate de strontium, d'action mixte, a été récemment retiré du marché en raison d'effets secondaires cardiovasculaires. À l'instar des chondrocytes arthrosiques, les ostéocytes de patients ostéoporotiques expriment également plusieurs marqueurs de sénescence [23]. Cette sénescence est également retrouvée dans les ostéocytes isolés de souris âgées souffrant d'ostéoporose [11]. À partir de ce constat, le groupe de $\mathrm{S}$. Khosla a utilisé le modèle de souris transgénique INK-ATTAC afin d'évaluer l'effet de l'élimination de ces cellules sénescentes sur l'architecture osseuse des souris ostéoporotiques âgées [11]. La sénolyse induite par l'administration per os d'AP20187 s'est traduite par une diminution du nombre d'ostéocytes sénescents, mais aussi d'ostéoclastes (les cellules responsables de la résorption osseuse). Ceci aboutit alors à une augmentation du volume de l'os trabéculaire (ou os spongieux) ou des vertèbres (visualisé par microCT ${ }^{7}$ ), un plus grand nombre de travées osseuses, et des propriétés biomécaniques améliorées des os de la souris âgée. Ces résultats démontrent le lien causal entre la présence de cellules sénescentes et la perte osseuse.

${ }^{6}$ Le denosumab est un anticorps monoclonal qui neutralise la molécule RANKL, ligand du récepteur RANK, exprimé par les ostéoclastes, empêchant leur activation.

${ }^{7}$ Microtomographie à rayons X. II s'agit d'une microscopie non-invasive radiographique tridimensionnelle. 


\section{Médicament sénolytique : définition et modes d'action}

À l'aide des modèles murins transgéniques que nous avons présentés, le lien entre l'état de sénescence dans certains types cellulaires d'un tissu donné et l'émergence de pathologies du sujet âgé a pu s'élargir à de nombreux organes tels que le foie, les poumons, les reins ou très récemment, le cerveau $[3,24]$. Ces travaux montrent qu'éliminer pharmaco-génétiquement les cellules sénescentes permet de s'opposer aux pathologies du vieillissement des organes étudiées. Ils sont ainsi un appel à développer des traitements dits «sénolytiques », c'està-dire capables de reproduire l'élimination spécifique des cellules sénescentes dans I'organisme, comme le fait la molécule AP20187 dans les souris INK-ATTAC ou le ganciclovir dans les 3MR. Un sénolytique peut alors se définir comme un traitement pharmacologique, ou autre, permettant l'élimination sélective des cellules sénescentes d'un tissu ou d'un organisme entier. Cette notion peut s'étendre également à tout médicament capable de réduire les effets délétères liés à la présence de cellules sénescentes. On parle alors de «sénothérapies» pour englober toutes ces approches fondées sur le ciblage spécifique des cellules sénescentes ou leurs effets délétères sur un tissu donné ou un organisme entier.

Trois principales stratégies ont été proposées pour sélectionner des molécules capables de cibler uniquement des cellules sénescentes, et non des cellules différenciées ou proliférantes. Les cellules sénescentes sont résistantes aux signaux pro-apoptotiques. Cela repose sur la surexpression par ces cellules de protéines impliquées dans des voies de signalisation de survie telles que l'inhibiteur du cycle cellulaire, p2 $1^{\text {wafl }}$, la protéine anti-apoptose $\mathrm{Bcl}-\mathrm{xl}$ (B-cell lymphoma-extra large), la protéine chaperone HSP90 (heat shock protein 90), la voie des kinases ERK (extracellular signal-regulated kinases), la protéine FOX04 (Forkhead Box 04), ou la production de facteurs de survie, incluant le plasminogenactivator inhibitor-1 (PAI1), CCL5 (C-C motif chemokine ligand 5) ou les éphrines ${ }^{8}$ EFNB-1 et 3 [25-28]. Le mode d'action des sénolytiques repose sur leur capacité d'inhiber ces protéines de survie afin de lever la résistance à l'apoptose. Le navitoclax, également appelé UBX0101, fut l'un des premiers composés dits «sénolytiques » à être décrit. Initialement développé pour cibler des cellules tumorales par son activité inhibitrice des protéines de la famille $\mathrm{Bcl}-2$, dont $\mathrm{Bcl}-\mathrm{xl}$, le navitoclax de la même manière que les autres molécules membres de cette famille d'inhibiteurs, est capable d'induire spécifiquement la mort cellulaire des fibroblastes sénescents et non de ceux qui prolifèrent [29-31]. In vivo, le traitement per os de souris irradiées par du Navitoclax a permis l'élimination des cellules sénescentes. Ce traitement aboutit alors à une diminution de l'expression des protéines sécrétoires du SASP et, au final, à une réduction des signes cliniques de plusieurs pathologies comme la fibrose pulmonaire [32]. De la même manière, dans un modèle de souris progérique XPD', la perturbation de l'interaction entre FOX04 et le suppresseur de tumeur p53 par l'utilisation d'un peptide interférent a

\footnotetext{
${ }^{8}$ Protéines transmembranaires assurant des interactions cellule-cellule par liaison à leurs récepteurs $\varepsilon p h$. ${ }^{9}$ Le syndrome xeroderma pigmentosum du groupe $D(X P D)$ est une maladie congénitale à transmission génétique autosomique récessive. II provoque une photosensibilité, un vieillissement accéléré, des défauts de stature, et une dégénérescence des tissus.
}

abouti à l'apoptose des cellules sénescentes, à une amélioration de la fonction rénale, ainsi qu'à une réversion du phénotype progéroïde [28]. On peut aussi citer l'association prometteuse de dasatinib et de quercétine. Le dasatinib est un pan-inhibiteur de tyrosine kinase (IKT). Ce produit est utilisé comme traitement de seconde ligne dans des pathologies onco-hématologiques [33]. II inhiberait les voies de signalisation dépendantes des éphrines [3]. La quercétine, elle, est un flavonoïde présent dans de nombreux végétaux. Elle est connue pour ses propriétés anti-oxydantes et anti-inflammatoires [25], ainsi qu'inhibitrice des serpines [3]. Cette combinaison innovante montrerait des propriétés synergiques d'induction de mort cellulaire sur des cellules endothéliales et des pré-adipocytes sénescents [25].

La deuxième stratégie "sénolytique » est fondée sur le ciblage des adaptations métaboliques opérées par les cellules sénescentes. L'utilisation d'inhibiteurs de ces voies métaboliques privilégiées pourrait être un moyen d'induire spécifiquement leur mort. L'équipe de CA Schmitt a ainsi pu montrer l'apparition d'un phénotype hypercatabolique et glycolytique par les cellules sénescentes sanguines, en réponse à une chimiothérapie, dans un modèle murin de lymphomes. L'administration de 2-déoxyglucose (2DG), un inhibiteur de la glycolyse, en combinaison avec cette chimiothérapie, a permis l'élimination complète de ces cellules sénescentes et a réduit le risque de rechute [34].

Enfin, la dernière approche permettant de cibler les cellules sénescentes serait de bloquer leurs effets paracrines et autocrines délétères sur le micro-environnement tissulaire. Il s'agit ici d'inhibiteurs pharmacologiques des voies de signalisation contrôlant positivement l'établissement du SASP. Parmi les molécules testées, on retrouve, en outre, le ruxolitinib, un inhibiteur de la voie des Janus kinases (JAK). Des souris âgées traitées chroniquement avec cette drogue ont une amélioration des paramètres de l'ostéoporose [11], démontrant l'intérêt thérapeutique de ce type de stratégies sur des pathologies du sujet âgé (Tableau I).

\section{Les sénolytiques : nouvelles perspectives thérapeutiques des pathologies ostéo- articulaires du sujet âgé}

À ce jour, aucun traitement médical curatif n'est disponible pour le traitement de l'arthrose. Les nouveaux «médicaments symptomatiques à action lente » proposés récemment, comme la glucosamine ou l'injection articulaire d'acide hyaluronique, n'ont pas encore montré des preuves d'efficacité sur le remodelage articulaire structurel à long terme [35]. Seuls des traitements anti-douleurs 


\begin{tabular}{|c|c|c|c|}
\hline Drogues & Cibles & Pathologies traitées & Réf. \\
\hline \multicolumn{4}{|c|}{ Approche pharmacogénétique } \\
\hline AP20187 & $\begin{array}{l}\text { Active la caspase } 8 \text { et la cascade conduisant à } \\
\text { l'apoptose dans le modèle murin INK-ATTAC }\end{array}$ & $\begin{array}{l}\text { Arthrose, ostéoporose, sarcopénie, } \\
\text { cataracte, arythmie et insuffisance } \\
\text { cardiaque, insuffisance rénale }\end{array}$ & $\begin{array}{l}{[10,11} \\
14,15]\end{array}$ \\
\hline Ganciclovir & $\begin{array}{l}\text { Inhibiteur de la tyrosine kinase du virus herpès } \\
\text { simplex } 1 \text { dans le modèle murin P16-3MR }\end{array}$ & Arthrose, ostéoporose & {$[10,11]$} \\
\hline \multicolumn{4}{|c|}{ Levée de la résistance à l'apoptose } \\
\hline Navitoclax & $\begin{array}{l}\text { Protéines anti-apoptotiques } \\
\mathrm{Bcl}-\mathrm{xl} \text { et } \mathrm{Bcl}-\mathrm{w}\end{array}$ & $\begin{array}{l}\text { Arthrose, fibrose pulmonaire, } \\
\text { athérosclérose }\end{array}$ & {$[10,13,32]$} \\
\hline Dasatinib + Quercetin & $\begin{array}{l}\text { D : pan-inhibiteur de tyrosine kinase, inhibiteur } \\
\text { de la voie de signalisation des éphrines } \\
Q: \text { inhibiteur des serpines }\end{array}$ & Ostéoporose & {$[11]$} \\
\hline Peptide F0X04-DRI & $\begin{array}{l}\text { Entraîne l'apoptose en empêchant l'interaction } \\
\text { entre F0X04 et p53 }\end{array}$ & Insuffisance rénale & {$[28]$} \\
\hline \multicolumn{4}{|c|}{ Modification du profil métabolique } \\
\hline 2-déoxyglucose & Inhibiteur de la glycolyse & Lymphome & {$[34]$} \\
\hline \multicolumn{4}{|l|}{ Inhibition du SASP } \\
\hline Ruxolitinib & Inhibiteur des janus kinases 1 et 2 & Ostéoporose & [11] \\
\hline
\end{tabular}

Tableau I. Liste des approches sénolytiques appliquées aux pathologies ostéo-articulaires du sujet âgé.

symptomatiques et la physiothérapie (dont la kinésithérapie) restent les principales alternatives avant le remplacement de l'articulation touchée par une prothèse lorsque cela est possible. Une réelle et efficace approche thérapeutique est donc très attendue par le monde médical et par les patients. Afin de tester l'efficacité de médicaments sénolytiques comme nouveau traitement innovant de l'arthrose, Jeon et al. ont réalisé, tous les 2 jours, des injections intra-articulaires de UBX0101/navitoclax chez des souris jeunes après section du ligament croisé, dès que la sénescence articulaire était détectable. Ce traitement a significativement réduit la présence de cellules sénescentes articulaires, la dégradation du cartilage et la douleur liée à l'arthrose. De plus, à l'instar des résultats obtenus avec les modèles murins transgéniques, le navitoclax réduit l'expression articulaire de MMP-13, d'IL-6 et d'IL-1 $\beta$, tout en favorisant la formation de néo-cartilage, avec une augmentation de la production de collagène de type II et d'aggrécane. Pour traduire ces résultats de modèles murins chez les patients atteints d'arthrose, ces auteurs offrent des preuves supplémentaires, en montrant que le traitement in vitro par navitoclax sur des explants de cartilage réduit l'expression des gènes associés à la sénescence tout en augmentant le taux de prolifération des chondrocytes non sénescents. En éliminant les cellules sénescentes, le navitoclax non seulement supprime un acteur causal dans le processus arthrosique, mais restaure également un environnement pro-chondrogénique. Ainsi, les auteurs ajoutent une nouvelle catégorie de médicaments prometteurs dans l'arsenal thérapeutique de tous les médecins impliqués dans la prise en charge de l'arthrose.
Comme pour toute nouvelle étape majeure en médecine, plusieurs questions devraient être résolues avant l'application humaine de ce type de sénolytique aux traitements de l'arthrose. Tout d'abord, le navitoclax est déjà utilisé comme traitement de divers cancers, dans des essais de phase I/II. Ces essais cliniques ont fait apparaître des effets secondaires importants, comme une thrombocytopénie ${ }^{10}$ [36]. Le choix d'injecter ce sénolytique en intra-articulaire diminuerait l'exposition systémique et, de fait, ses potentiels effets secondaires. Pour extrapoler les résultats expérimentaux aux patients, les auteurs ont également traité des souris âgées souffrant d'arthrose spontanée. Dans ce cas, l'injection intra-articulaire de navitoclax seul n'a présenté aucun effet sur la dégradation du cartilage et n'a pas amélioré l'expression des gènes chondrogéniques, à la différence de ce qui avait été observé avec des souris jeunes. En revanche, l'élimination systémique des cellules sénescentes, par des traitements d'une semaine chaque mois, à partir du douzième mois de vie jusqu'à leur mort naturelle, a nettement réduit la dégradation spontanée du cartilage liée à l'âge, confirmant que cibler la sénescence était également une voie prometteuse chez les patients âgés.

\footnotetext{
${ }^{10}$ Diminution du nombre de plaquettes sanguines.
} 
L'injection intra-articulaire chez les patients arthrosiques ne serait donc probablement pas suffisante et un traitement systémique devrait être envisagé. Le rapport bénéfice/risque de l'administration systémique de thérapies anticancéreuses pour retarder l'apparition de l'arthrose nécessite ainsi d'être soigneusement étudié et accepté par le patient. L'arthrose n'est pas la seule pathologie rhumatologique dégénérative à pouvoir espérer bénéficier des traitements sénolytiques. L'ostéoporose compte également parmi ces pathologies. Les médicaments inhibiteurs de la résorption osseuse, comme les bisphosphonates ou le denosumab, très fréquemment prescrits, souffrent d'une très mauvaise réputation auprès des patients ostéoporotiques, entraînant une faible adhérence thérapeutique. II y a donc une vraie nécessité médicale de découvrir de nouveaux traitements pour traiter cette pathologie très répandue. Après les résultats prometteurs de l'approche pharmacogénétique consistant en l'élimination des ostéoclastes sénescents dans les souris transgéniques INK-ATTAC, la combinaison associant le dasatinib et la quercétine a été testée. L'administration per os intermittente de ces deux principes actifs (l gavage, 1 fois par mois, pendant 4 mois) à des souris ostéoporotiques de 20 mois, montre une diminution des ostéocytes sénescents, une réduction de l'expression de la protéine $\mathrm{pl} 6^{\mathrm{Ink} 4 a}$, mais aussi et surtout une amélioration significative de l'architecture osseuse trabéculaire et corticale [11]. Comme précisé plus haut, ces mêmes souris âgées, traitées cette fois-ci chroniquement pendant 2 mois avec le ruxolitinib, un inhibiteur des kinases JAK1 et JAK2, à l'origine du SASP, montrent une amélioration tout à fait similaire des paramètres osseux trabéculaires et corticaux tout en réduisant le nombre d'ostéoclastes. C'est donc par la sécrétion du SASP que les cellules sénescentes exercent leurs effets délétères sur les os en favorisant l'émergence d'ostéoclastes [11].

Bien que toutes ces approches sénolytiques, développées chez la souris, ne ciblent pas spécifiquement les ostéocytes sénescents mais l'intégralité des cellules sénescentes des animaux traités, cibler par sénothérapie ces cellules chez une souris âgée souffrant d'ostéoporose, permet de réduire les signes cliniques de la maladie, ouvrant des pistes thérapeutiques innovantes.

\section{Une révolution médicale en marche pour la prise en charge des sujets âgés}

Les évidences s'accumulent pour établir un lien physiopathologique entre présence de sénescence cellulaire et apparition des pathologies liées à l'âge, en particulier ostéo-articulaires. Les sénothérapies constituent une révolution médicale dans la prise en charge des sujets âgés, en offrant des solutions thérapeutiques nouvelles, en particulier pour soigner l'arthrose et l'ostéoporose. Néanmoins, la sénescence cellulaire est un processus physiologique qui est bénéfique à l'organisme par son implication dans la cicatrisation et la suppression tumorale [2]. Nous manquons ainsi de recul sur les effets à long terme et l'innocuité de ces traitements sénolytiques sur la santé humaine. De nombreux travaux sont encore nécessaires; il est notamment essentiel de déterminer les signatures omiques caractéristiques des cellules sénescentes de différents tissus et en réponse à différents types de signaux inducteurs. Cela permettra de définir les séno- lytiques présentant le moins d'effets secondaires possibles et qui soient adaptés aux traitements de pathologies bien définies. AgeMed, I'un des deux premiers programmes transversaux nationaux regroupant 13 équipes de l'Inserm, qui a été initié en 2018, a justement cette ambition : identifier et proposer des cibles pour de futurs sénolytiques en comparant différents types cellulaires dont la sénescence a été induite. Ces sénothérapies ciblées pourraient être appliquées à des sujets âgés, sous la forme de cures relativement espacées et même localisées à un type d'organe comme une articulation malade, laissant alors la possibilité à d'autres cellules sénescentes, ailleurs dans l'organisme, d'exprimer leurs propriétés bénéfiques. $\diamond$

\section{SUMMARY}

Senescent cells: a target in osteo-articular diseases Our societies are facing with the emergence of an exponential number of patients with age-related degenerative chronic diseases such as osteoarthritis or osteoporosis. The "better" aging will thus be at the center of the next medical challenges in order to delay the loss of independence of the elderly and reduce costs of our health services. Over the last 5 years, based on innovative mouse models or in vitro studies, several research teams have demonstrated that many age-related degenerative diseases have in common a deleterious accumulation of so-called senescent cells in their respective deficient tissues. Thus, under the concept of senolysis, it has been proposed to target pharmacologically in vivo these cells to eliminate them and thus delay the emergence of these chronic diseases of the elderly subject. We propose here to summarize the recent strategies applied for the identification of novel senolytics and their uses in osteoarthritis and osteoporosis therapies $\diamond$

\section{REMERCIEMENTS}

Nous remercions Giuseppe Fusi pour sa relecture et correction du manuscrit.

Une dotation Inserm-projet transversal a été attribuée à l'équipe de C. Jorgensen dans le cadre du projet AgeMed.

\section{LIENS D'INTÉRÊT}

Les auteurs déclarent n'avoir aucun lien d'intérêt concernant les données publiées dans cet article.

\section{RÉFÉRENCES}

1. Hayflick L, Moorhead PS. The serial cultivation of human diploid cell strains. Exp Cell Res $1961 ; 25:$ 585-621.

2. He S, Sharpless NE. Senescence in health and disease. Cell $2017 ; 169: 1000$ 11.

3. Childs BG, Gluscevic M, Baker DJ, et al. Senescent cells: an emerging target for diseases of ageing. Nat Rev Drug Discov 2017 ; 16 : 718-35.

4. Coppe JP, Patil CK, Rodier F, et al. Senescence-associated secretory phenotypes reveal cell-nonautonomous functions of oncogenic RAS and the p53 tumor suppressor. PLoS Biol 2008 ; 6 : 2853-68. 


\section{RÉFÉRENCES}

5. Muñoz-Espín D, Serrano M. Cellular senescence: from physiology to pathology. Nat Rev Mol Cell Biol $2014 ; 15: 482-96$.

6. Hernandez-Segura A, Nehme J, Demaria M. Hallmarks of cellular senescence. Trends Cell Biol 2018.

7. Vicente R, Mausset-Bonnefont AL, Jorgensen C, et al. Cellular senescence impact on immune cell fate and function. Aging Cell $2016 ; 15: 400-6$.

8. Demaria M, Ohtani N, Youssef SA, et al. An essential role for senescent cells in optimal wound healing through secretion of PDGF-AA. Dev Cell 2014 ; 31 : 722-33.

9. Yun MH, Davaapil H, Brockes JP. Recurrent turnover of senescent cells during regeneration of a complex structure. Elife $2015 ; 4$

10. Jeon $\mathrm{OH}, \mathrm{Kim} \mathrm{C}$, Laberge RM, et al. Local clearance of senescent cells attenuates the development of post-traumatic osteoarthritis and creates a pro-regenerative environment. Nat Med 2017; 23 775-81

11. Farr JN, Xu M, Weivoda MM, et al. Targeting cellular senescence prevents age-related bone loss in mice. Nat Med 2017 ; $23: 1072-9$.

12. García-Prat L, Martínez-Vicente M, Perdiguero $\varepsilon$, et al. Autophagy maintains stemness by preventing senescence. Nature $2016 ; 529: 37-42$.

13. Childs BG, Baker DJ, Wijshake T, et al. Senescent intimal foam cells are deleterious at all stages of atherosclerosis. Science 2016 ; $354: 472-7$.

14. Baker DJ, Childs BG, Durik M, et al. Naturally occurring pl6(Ink4a)-positive cells shorten healthy lifespan. Nature 2016 ; 530 : 184-9.

15. Baker DJ, Wijshake T, Tchkonia T, et al. Clearance of pl6Ink4a-positive senescent cells delays ageing-associated disorders. Nature $2011 ; 479$ : 232-6.

16. Lawrence RC, Felson DT, Helmick CG, et al. Estimates of the prevalence of arthritis and other rheumatic conditions in the United States. Part II. Arthritis Rheum $2008 ; 58: 26-35$.

17. Scanzello CR, Goldring SR. The role of synovitis in osteoarthritis pathogenesis. Bone $2012 ; 51: 249-57$.

18. $Y_{u} \mathrm{D}, \mathrm{Xu}$ J, Liu F, et al. Subchondral bone changes and the impacts on joint pain and articular cartilage degeneration in osteoarthritis. Clin Exp Rheumatol 2016 ; 34 : 929-34.

19. Cooper C, Campion G, Melton LJ. Hip fractures in the elderly: a world-wide projection. Osteoporos Int $1992 ; 2: 285-9$.

20. Bass $\varepsilon$, French DD, Bradham DD, Rubenstein LZ. Risk-adjusted mortality rates of elderly veterans with hip fractures. Ann Epidemiol $2007 ; 17$ : 514-9.

21. Khosla S, Burr D, Cauley J, et al. Bisphosphonate-associated osteonecrosis of the jaw: report of a task force of the American society for bone and mineral research.J Bone Miner Res $2007 ; 22$ : 1479-91.

22. Shane $\varepsilon$, Burr D, Abrahamsen B, et al. Atypical subtrochanteric and diaphyseal femoral fractures: second report of a task force of the American society for bone and mineral research. J Bone Miner Res $2014 ; 29: 1-23$.

23. Farr JN, Fraser DG, Wang $\mathrm{H}$, et al. Identification of senescent cells in the bone microenvironment.J Bone Miner Res 2016 ; $31: 1920$-9.

24. Chinta SJ, Woods G, Demaria M, et al. Cellular senescence is induced by the environmental neurotoxin paraquat and contributes to neuropathology linked to Parkinson's disease. Cell Rep $2018 ; 22: 930-40$.
25. Zhu Y, Tchkonia T, Pirtskhalava T, et al. The Achilles' heel of senescent cells: from transcriptome to senolytic drugs. Aging Cell $2015 ; 14: 644-58$.

26. Fuhrmann-Stroissnigg H, Ling Yy, Zhao J, et al. Identification of HSP90 inhibitors as a novel class of senolytics. Nat Commun $2017 ; 8: 422$.

27. Kochetkova EY, Blinova GI, Bystrova OA, et al. Targeted elimination of senescent Ras-transformed cells by suppression of MEK/ERK pathway. Aging (Albany NY) 2017 ; $9: 2352-75$.

28. Baar MP, Brandt RMC, Putavet DA, et al. Targeted apoptosis of senescent cells restores tissue homeostasis in response to chemotoxicity and aging. Cell 2017 ; 169 : 132-47.el6.

29. Chang J, Wang $Y$, Shao L, et al. Clearance of senescent cells by ABT263 rejuvenates aged hematopoietic stem cells in mice. Nat Med 2016 ; 22 : 78-83.

30. Yosef R, Pilpel N, Tokarsky-Amiel R, et al. Directed elimination of senescent cells by inhibition of BCL-W and BCL-XL. Nat Commun $2016 ; 7$ : 11190 .

31. Zhu Y, Tchkonia T, Fuhrmann-Stroissnigg $\mathrm{H}$, et al. Identification of a novel senolytic agent, navitoclax, targeting the $\mathrm{Bcl}-2$ family of anti-apoptotic factors. Aging Cell $2016 ; 15: 428-35$.

32. Pan J, Li D, Xu Y, et al. Inhibition of Bcl-2/XI with ABT-263 selectively kills senescent type II pneumocytes and reverses persistent pulmonary fibrosis induced by ionizing radiation in mice. Int J Radiat Oncol Biol Phys 2017 ; 99 : 353-61.

33. Talpaz M, Shah NP, Kantarjian $\mathrm{H}$, et al. Dasatinib in imatinib-resistant Philadelphia chromosome-positive leukemias. N EnglJ Med 2006 ; 354 : 2531-41.

34. Dörr JR, Yu Y, Milanovic M, et al. Synthetic lethal metabolic targeting of cellular senescence in cancer therapy. Nature 2013 ; 501 : 421-5.

35. McAlindon TE, Bannuru RR, Sullivan MC, et al. OARSI guidelines for the nonsurgical management of knee osteoarthritis. Osteoarthritis Cartilage 2014 ; $22: 363-88$.

36. Wilson WH, O'Connor OA, Czuczman MS, et al. Navitoclax, a targeted highaffinity inhibitor of $B C L-2$, in lymphoid malignancies: a phase 1 doseescalation study of safety, pharmacokinetics, pharmacodynamics, and antitumour activity. Lancet Oncol $2010 ; 11$ : 1149-59.

37. Goy $\varepsilon$, Abbadie C. Sénescence et cancer : double jeu. Med Sci (Paris) 2018 ; $34: 223-30$.

38. Chauvet V, Jouaville S, Garbez N, Martins I. L'élimination des cellules sénescentes : vers un avenir meilleur? Med Sci (Paris) 2016; 32 : 1030-3.

\section{Bon de commande à retourner à EDP Sciences, 17, avenue du Hoggar, 91944 Les Ulis Cedex A Tél. : 0149856069 - Fax : 0149850345 - E-mail : francois.flori@edpsciences.org}

TIRÉS À PART

J.M. Brondello

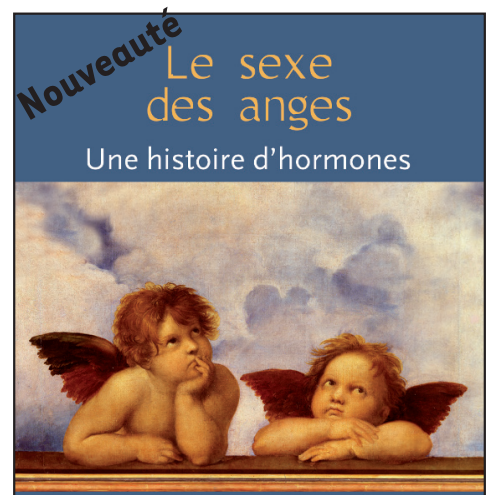

NATHALIE JOSSO

Illustrations Martine Netter
NOM :

Prénom :

Adresse :

Code postal :

Ville :

Pays :

Fonction :

Je souhaite recevoir l'ouvrage

Le sexe des anges : $20 €+3 €$ de port $=23 €$ TTC

en

exemplaire, soit un total de

Par chèque, à l'ordre de EDP Sciences

Par carte bancaire :

$\square$ Visa

Eurocard/Mastercard

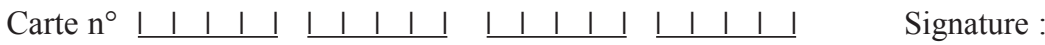

Date d'expiration :

$\mathrm{N}^{\circ}$ de contrôle au dos de la carte :

iil Inserm 